Abstract 20 Table 1 Univariable and ultivariable hazard ratios for follow-up mortality

\begin{tabular}{lll}
\hline & $\begin{array}{l}\text { Unadjusted HR }(95 \% \mathrm{Cl}) \\
(\mathbf{n = 3 2 2 2})\end{array}$ & $\begin{array}{l}\text { Adjusted HR }(95 \% \mathrm{Cl}) \\
(\mathbf{n}=3222)\end{array}$ \\
\hline Age & 1.021 .021 .02 & 1.031 .021 .03 \\
Gender (F vs M) & 0.920 .831 .01 & 0.820 .740 .91 \\
White & 1.00 & 1.00 \\
South Asia & 0.810 .710 .93 & 0.830 .690 .99 \\
Black & 0.650 .540 .78 & 0.980 .861 .13 \\
Other Asia & 0.740 .600 .91 & 0.840 .701 .02 \\
Background & & 0.930 .751 .14 \\
Mixed/Chinese/ & 0.700 .590 .84 & \\
Unknown & & \\
\hline
\end{tabular}

communities. Large-scale studies examining real world cohorts and factors that affect their outcomes are lacking.

Design A retrospective cohort study of 3626 patients admitted with a diagnosis of heart failure over a 9-year period. We investigated the effects of baseline characteristics, co-morbidities and echocardiographic findings on in-hospital and overall mortality along with hospital readmission. Mean ages were 74.8 and 78.7 years for males and females respectively. The average follow-up time was 3 years for mortality and 2.43 years for readmission.

Results Increasing age is associated with higher mortality rate both in-hospital OR 1.04 [1.03-1.05 95\% CI] and throughout the follow-up period (overall mortality) HR 1.03 [1.02-1.03 95\% CI]. Being female had a protective effect for overall mortality HR 0.82 [0.74-0.91 95\% CI]. (Table 1). Ethnicity had a mixed effect. For in-hospital mortality, there was an adverse association for Mixed Asian OR 1.5 [1.00-2.27 95\% $\mathrm{CI}]$ and Chinese backgrounds OR 1.53 [1.11-2.13 95\% CI]. For readmission, there was significant variation amongst different ethnic groups - Black patients were at highest risk HR $1.25[1.02-1.5495 \% \mathrm{CI}]$ in contrast to Chinese patients who had the lowest risk HR 0.68 [0.53-0.86 95\% CI]. (Table 2).

Subgroup analysis of echocardiography data demonstrated that compared to patients with severe systolic dysfunction (LVEF $<35 \%)$, patients at all other stages of systolic dysfunction, including Heart Failure with Preserved Ejection Fraction (LVEF $>60 \%)$, did not have any difference in mortality. This remained true for both in-hospital (0.86 OR [0.24-3.05 95\% $\mathrm{CI}]$ and overall mortality $1.19 \mathrm{HR}$ [0.77-1.83 95\% CI].

\begin{tabular}{lll}
$\begin{array}{l}\text { Abstract } 20 \\
\text { for follow-up readmission }\end{array}$ & \multicolumn{1}{c}{ Univariable and multivariable hazard ratios } \\
\hline & $\begin{array}{l}\text { Unadjusted HR }(95 \% \mathrm{Cl}) \\
(\mathbf{n}=3222)\end{array}$ & $\begin{array}{l}\text { Adjusted } \mathrm{HR}(95 \% \mathrm{Cl} 95 \% \mathrm{Cl}) \\
(\mathbf{n}=3222)\end{array}$ \\
\hline Age & 1.011 .001 .01 & 1.011 .001 .02 \\
Gender (F vs M) & 1.000 .881 .13 & 0.970 .861 .11 \\
White & 1.00 & 1.00 \\
South Asia & 0.960 .721 .30 & 1.030 .871 .22 \\
Black & 0.740 .451 .22 & 1.251 .021 .54 \\
Other Asia & 0.600 .351 .05 & 1.050 .821 .34 \\
Background & 0.500 .300 .86 & 0.680 .530 .86 \\
Mixed/Chinese/ & & \\
Unknown & &
\end{tabular}

Conclusion In a real world heart failure population, we have demonstrated novel associations between ethnicity and disease trajectory and confirmed that LVEF is a poor prognostic marker. Future work is planned to assess why ethnic groups may have different outcomes, including examination of patient understanding and engagement with healthcare.

\section{IMPACT OF COMBINED ATRIAL FIBRILLATION AND HEART FAILURE ON MORTALITY: 14 YEAR NATURALISTIC FOLLOW-UP STUDY}

${ }^{1}$ Oliver Ziff*, ${ }^{2}$ Paul R Carter, ${ }^{3}$ John McGowan, ${ }^{4}$ Suresh Chandran, ${ }^{5}$ Hardeep Uppal, ${ }^{6}$ Rahul Potluri. 'University of Birmingham; ${ }^{2}$ Royal Free London NHS Foundation Trust; ${ }^{3}$ Central Manchester University Foundation Trust; ${ }^{4}$ Department of Acute Medicine, North Western Deanery; ${ }^{5}$ ACALM Study Unit in Collaboration with Aston Medical School; ${ }^{6}$ ACALM Study Unit in Collaboration with Aston Medical School, Aston University; *Presenting Author

\subsection{6/heartjnl-2016-309890.21}

Background Atrial Fibrillation (AF) and Heart Failure (HF) frequently co-exist conferring considerable morbidity and mortality, yet current treatment options remain limited. Recent meta-analyses of patients with concomitant $\mathrm{AF}$ and $\mathrm{HF}$ have suggested no prognostic benefit of beta-blockers or digoxin, creating a paradox whereby those most in need have the fewest therapeutic choices. We sought to investigate the association between $\mathrm{HF}$ and $\mathrm{AF}$ and their impact on mortality from a large 14-year naturalistic follow-up study.

Methods Anonymous data of adult patients aged $\geq 18$ with all types of HF and AF admitted to several hospitals in the North of England between 2000 and 2013 was obtained and processed using the ACALM (Algorithm for Co-morbidity, Associations, Length of stay and Mortality) study protocol. ACALM uses the ICD-10 and OPCS-4 coding systems to identify patients and the methodology has been published widely. Analyses were performed comparing mortality between patients with $\mathrm{HF}, \mathrm{AF}$ and combined $\mathrm{HF}$ and $\mathrm{AF}$ at baseline and their development during follow-up.

Results At baseline, of 929,552 adult patients 29,164 (3.1\%) had AF, 19,474 (2.1\%) had HF, and 5,728 (0.6\%) had both $\mathrm{HF}$ and AF. Of those with $\mathrm{AF}$ at baseline, 1,647 (5.6\%)

5 year survival for Atrial Fibrillation (AF) and Heart Failure (HF) patients

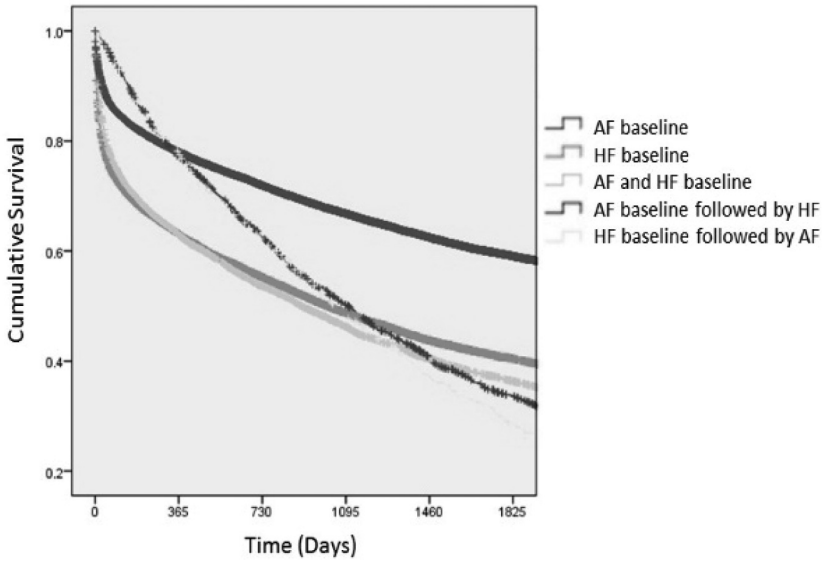

Abstract 21 Figure 1 Kaplan Meier survival curves through the duration of follow-up in the ACALM database are shown based on atrial fibrillation and heart failure status at the start and end of the study. AF, atrial fibrillation; HF, heart failure 


\begin{tabular}{|c|c|c|c|c|c|}
\hline & $\begin{array}{l}\text { Baseline } \\
\text { AF }\end{array}$ & $\begin{array}{l}\text { Baseline } \\
\text { HF }\end{array}$ & $\begin{array}{l}\text { BaselineAF } \\
+\mathrm{HF}\end{array}$ & $\begin{array}{l}\text { Baseline AF, } \\
\text { developed HF }\end{array}$ & $\begin{array}{l}\text { Baseline } \mathrm{HF}, \\
\text { developed AF }\end{array}$ \\
\hline $\mathrm{n}$ & 29164 & 19474 & 5728 & 1647 & 824 \\
\hline Age $\pm S D$ & $74 \pm 13$ & $73 \pm 14$ & $77 \pm 12$ & $77 \pm 11$ & $77 \pm 11$ \\
\hline Male\% & 52.4 & 51.0 & 49.8 & 48.0 & 49.3 \\
\hline Caucasian\% & 87.6 & 82.9 & 86.8 & 92.2 & 90.2 \\
\hline South Asian\% & 1.7 & 4.0 & 1.8 & 1.6 & 2.4 \\
\hline $\begin{array}{l}\text { Afro-Caribbean } \\
\%\end{array}$ & 0.8 & 1.6 & 1.2 & 0.4 & 0.6 \\
\hline Other\% & 9.9 & 11.8 & 10.2 & 5.8 & 6.8 \\
\hline $\begin{array}{l}\text { Crude } \\
\text { Mortality (per } \\
1000 \text { ) }\end{array}$ & 485 & 636 & 672 & 715 & 778 \\
\hline $\begin{array}{l}\text { Mean Survival } \\
\text { (Days) }\end{array}$ & 722 & 631 & 692 & 880 & 922 \\
\hline
\end{tabular}

$\mathrm{AF}$, atrial fibrillation; $\mathrm{HF}$, heart failure; $\mathrm{SD}$, standard deviation

developed HF during follow-up, and of those with $\mathrm{HF}$ at baseline, 824 (4.2\%) developed AF during follow-up. Demographics and crude mortality rates are shown; see Table. Patients with combined $\mathrm{AF}$ and $\mathrm{HF}$ at baseline had increased mortality than patients with $\mathrm{AF}$ or $\mathrm{HF}$ alone. Patients with $\mathrm{AF}$ at baseline that developed HF, and patients with HF at baseline that developed AF, experienced a greater mortality compared to those with combined $\mathrm{HF}$ and $\mathrm{AF}$ at baseline; see Figure.

Conclusion Concomitant AF and HF is associated with substantial mortality and risk of death, irrespective of which disease develops first. In light of limited current treatment for these patients, future therapies to specifically target the combined $\mathrm{HF}$ and $\mathrm{AF}$ group are required.

\section{AUDIT OF MANAGEMENT OF PATIENTS WITH HEART FAILURE FOLLOWING NON-STEMI AND USE OF ALDOSTERONE ANTAGONISTS}

${ }^{1}$ Olivia Buckledee*, ${ }^{2}$ Yasmin Ismail, ${ }^{2}$ Angus Nightingale. ${ }^{1}$ University of Bristol; ${ }^{2}$ Bristol Heart Institute; *Presenting Author

\subsection{6/heartjnl-2016-309890.22}

Background The presence of heart failure following a non-ST elevation myocardial infarction (non-STEMI) is associated with a worse prognosis. Current UK and European Guidelines recognise the importance of identifying these higher risk patients and offering them disease modifying therapies including drugs and devices.

We wanted to find out of these guidelines were being followed in a busy Heart Attack Centre in the South West of England; in particular, whether a mineralocorticoid receptor antagonist (MRA) was being prescribed to those with impaired LV function and either diabetes or signs heart failure.

Methods We performed a retrospective audit of consecutive patients identified as having non-STEMI on the MINAP database form April 2014 to March 2015.

We assessed:

1. How many received an echocardiogram during their index admission;
2. Compliance with NICE recommended post MI medications on discharge;

3. Implications for device implantation rates according to NICE and ESC guidelines.

Results 176 patients (mean age 70 years, 64\% male) were recorded on the MINAP database with non-STEMI in the study period. $71 \%(125 / 176)$ had an echocardiogram during the index admission. 42\% (53/125) had good left ventricular (LV) function and $30 \%(38 / 125)$ had moderately or severely impaired LV function ( $\mathrm{EF}<40 \%)$.

Of those with impaired LV function post non-STEMI, 82\% (31/38) either had diabetes or signs of pulmonary oedema; 6 of these had a documented contra-indication to a MRA. 56\% $(14 / 25)$ were prescribed a MRA in accordance with the NICE guidelines compared to $>95 \%$ compliance with guidelines recommending ACEi/BB/statin/antiplatelet agents. Mean length of stay for those with impaired LV function was 7.9 days. $71 \%$ (27/38) of the impaired LV function patients were followed up by our cardiology team with 11 referred back to their local hospital for follow up. $41 \%(11 / 27)$ had a repeat echo after at least one month to reassess LV function and assess requirement for implantable devices.

Conclusions Nearly a third of patients had significantly impaired LV function following non-STEMI. Whilst most postMI drugs were prescribed $>95 \%$ in compliance with the NICE guidelines, MRAs (spironolactone and eplerenone) were only prescribed in around half of suitable cases. The reasons for this could include poor documentation of contraindication to MRA or lack of awareness of the guidelines.

Follow-up reassessment of LV function after at least a month to detect functional recovery or indication for device therapy was also poor. Opportunities to prevent sudden death or worsening heart failure by implanting ICD or CRT devices could therefore have been missed.

Management of non-STEMI patients complicated by heart failure might benefit from a closer link between the acute coronary syndrome and the heart failure teams to improve outcomes and reduce length of stay for patients.

\section{SURVEILLANCE AND INCIDENCE OF CHEMOTHERAPY- INDUCED CARDIOTOXICITY IN BREAST CANCER: A LONG TERM OBSERVATIONAL STUDY}

Bayan Soujeri*, Jagdeep Singh, Samuel Chew, Sean Hawkey, Michelle Ferguson, Chim C Lang. University of Dundee; *Presenting Author

\subsection{6/heartjnl-2016-309890.23}

Introduction Recent advances in chemotherapy have transformed breast cancer from a fatal disease to a survivable condition. However, many patients who survive their cancer, succumb to the unintended adverse effects of therapy. Chemotherapy-induced cardiotoxicity is now among the most feared adverse effects and has been reported in up to $50 \%$ of patients up to twenty years later, the majority of which occurring after completion of therapy. Consequently, current breast cancer treatment guidelines recommend assessment of LV function before, during and after chemotherapy.

Methods We conducted a retrospective cohort study of 1263 breast cancer patients who received either anthracyclines alone or combined with the monoclonal antibody trastuzumab at Ninewells Hospital, Dundee between January 2003 and December 2014. Imaging modalities such as MUGA scans 